\title{
THE EXISTENCE AND UNIQUENESS OF WEAK SOLUTIONS FOR PRECIPITATION FRONTS: A NOVEL HYPERBOLIC FREE BOUNDARY PROBLEM IN SEVERAL SPACE VARIABLES
}

\author{
ANDREW J. MAJDA ${ }^{(1,2)}$ AND PANAGIOTIS E. SOUGANIDIS ${ }^{(3,4)}$
}

\begin{abstract}
The determination of the large scale boundaries between moist and dry regions is an important problem in contemporary meteorology. These phenomena have been addressed recently in a simplified tropical climate model through a novel hyperbolic free boundary formulation yielding three families (drying, slow moistening, and fast moistening) of precipitation fronts. The last two wave types violate Lax's shock inequalities yet are robustly realized. This formal hyperbolic free boundary problem is given here a rigorous mathematical basis by establishing the existence and uniqueness of suitable weak solutions arising in the zero relaxation limit. A new $L^{2}$-contraction estimate is also established at positive relaxation values.
\end{abstract}

\section{INTRODUCTION}

The goal here is to prove the existence and uniqueness of weak solutions to a novel hyperbolic free boundary value problem in several space variables that has emerged recently in the analysis of precipitation fronts in the large scale tropical atmosphere ([FMP], [SM], [PFM], [KM1], [KM2]). Precipitation fronts are the boundaries between the zones of extremely moist air (with constant precipitation) such as over the Indonesian marine continent, the Indian ocean, and Western Pacific, and the zones of extremely dry air in the tropics and subtropics that occur over areas such as the Galapagos islands at the equator or the Arabian peninsula in the subtropics. An important practical question in contemporary meteorology for long range weather prediction and climate change projections is what determines the boundaries of the precipitating fronts as well as their evolution in time. Such assessments are performed, for example, by the Intergovermental Panel for Climate Change (IPCC) by running extremely complex general computer models called GCM's. An important practical issue with the GCM's is how they treat moisture and what type of moisture waves do they

\footnotetext{
(1) Department of Mathematics and Climate Atmosphere Ocean Science, Courant Institute, New York University, 251 Mercer Street, New York, NY 10012, USA email: jonjon@math.cims.edu.

(2) Partially supported by the National Science Foundation and the Office of Naval Research.

(3) Department of Mathematics, The University of Chicago, 5734 S. University Avenue, Chicago, IL 60637, USA

email: souganidis@math.uchicago.edu.

(4) Partially supported by the National Science Foundation.
} 
produce at large scales compared with those in nature. This is very subtle. Although the GCM's have millions of variables and run on the largest supercomputers, they still have grid spacings of order $50 \mathrm{~km}$ to $200 \mathrm{~km}$. In addition many complex physical processes need to be parametrized often by adhoc recipes guided by physical intuition. These issues are discussed in detail in $[\mathrm{FMP}],[\mathrm{SM}],[\mathrm{PFM}]$, and the references there.

A novel mathematical theory of precipitating fronts was put forward in [FMP], [SM], $[\mathrm{PFM}]$ to address the above issues in idealized tropical climate models consisting of a shallow water system for the temperature, $T$, and velocity, $\mathbf{u}=(u, v)$, coupled with an equation for the moisture or humidity, $q$, through a relaxation source term $P$ representing the depletion of moisture through precipitation and the condensational heating of the atmosphere in making clouds and depending on the type of moisture parametrization. (See [FMP] for a detailed derivation).

The models are given by the hyperbolic system

$$
\left\{\begin{array}{l}
\mathbf{u}_{t}+\beta(y) \mathbf{u}^{\perp}=\nabla T, \\
T_{t}-\operatorname{div} \mathbf{u}=P, \quad \text { in } \quad \mathbb{R}^{2} \times(0, \infty), \\
q_{t}+\bar{Q} \operatorname{div} \mathbf{u}=-P,
\end{array}\right.
$$

where the constant $\bar{Q}$ in (0.1) represents the background moisture stratification of the atmosphere, and $\mathbf{u}^{\perp}=(-v, u)$, with initial conditions

$$
\mathbf{u}=\mathbf{u}_{0}, \quad T=T_{0}, \quad q=q_{0} .
$$

It is assumed here that

$$
\text { the rotation coefficient } \beta: \mathbb{R} \rightarrow \mathbb{R} \text { is smooth and constant for large }|y|
$$

and the source term $P$ has the structural form

$$
P=\varepsilon^{-1} \phi(q-\alpha T-\hat{q})
$$

where $\hat{q}$ is a saturation threshold constant, $\alpha$ is another constant reflecting different ways to parametrize the moisture process in clouds ([FMP], $[\mathrm{SM}],[\mathrm{PFM}]), \phi: \mathbb{R} \rightarrow[0, \infty)$ is a smooth approximation to $r_{+}=\max (r, 0)$, i.e., for some $r_{0}>0$,

$\phi$ is Lipschitz continuous, vanishes in $(-\infty, 0), \phi>0$ in $(0, \infty)$, and $\phi(r)=r$ for $r \geq r_{0}$.

The energy principles for well- posedness developed in [FMP] lead to the requirements

$$
0<\bar{Q}<1 \quad \text { and } 0<\alpha+\bar{Q} .
$$


Note that, based on additional physical reasoning (see $[\mathrm{FMP}],[\mathrm{SM}],[\mathrm{PFM}]$ and the references therein), contemporary GCM's often use either $\alpha=0$ or $\alpha=1$ and assume that the value of $\varepsilon$ is frozen to give a time scale between 2 and 12 hours.

A novel point of view for atmospheric science developed in $[\mathrm{FMP}],[\mathrm{SM}],[\mathrm{PFM}]$ is to formally take the zero relaxation limit, $\varepsilon \rightarrow 0$, in (0.1) and to study the type of the emerging precipitation fronts in order to get analytic insight into the behavior at positive $\varepsilon$. This procedure shows formally that, in the limit $\varepsilon \rightarrow 0$, precipitation fronts are free boundaries where $U=(\mathbf{u}, T, q)$ is continuous across them but $\nabla U$, which formally solves the hyperbolic system derived from (0.1), has jumps satisfying the Rankine-Hygoniot-type shock conditions $([\mathrm{M}])$. These considerations were utilized in $[\mathrm{FMP}]$ to build three distinct wave families, namely drying, slow moistening, and fast moistening precipitating fronts, with the last two families violating Lax's shock conditions. Nevertheless, careful numerical experiments demonstrate (see [FMP], [KM1], [KM2]) and additional mathematical theory (see [SM]) established, at positive $\varepsilon$, the robust realizability of all three wave types as well as interesting half smooth traveling waves. Finally numerical simulations (see [PFM]), again at $\varepsilon$ positive, confirm a theory for reflection and transmission of waves impinging on precipitation zones.

Given all the above mentioned formal results it is extremely interesting to pass to the zero relaxation limit in (0.1) and to prove rigorously the existence and uniqueness of suitable weak solutions for the limiting problem. This is the main topic of this note.

The paper is organized as follows: The first part of Section 1 contains a preliminary discussion of (0.1) at $\varepsilon$ positive including energy estimates for first derivatives and a new $L^{2}$-contraction property. In Section 2 we prove the existence of weak solutions of the problem obtained at the limit $\varepsilon \rightarrow 0$ and we develop an intrinsic characterization of the problem as a variational inequality. The proof of the uniqueness of the weak solutions is established in Section 3. Concluding remarks are presented in Section 4.

\section{Preliminary energy estimates and $L^{2}$-COntractions}

After some straightforward algebraic manipulations (0.1) can be written in the more convenient form

$$
\left\{\begin{array}{l}
\mathbf{u}_{t}+\beta(y) \mathbf{u}^{\perp}=\nabla T, \\
(q+T)_{t}-(1-\bar{Q}) \operatorname{div} \mathbf{u}=P, \quad \text { in } \mathbb{R}^{2} \times(0, \infty), \\
(q-\alpha T-\hat{q})_{t}+(\bar{Q}+\alpha) \operatorname{div} \mathbf{u}=-(1-\alpha) P .
\end{array}\right.
$$

For the interpretation of the results below it is convenient to introduce two new variables, namely the equivalent temperature, $T_{e}$, and the equivalent moisture, $q_{e}$, given respectively 
by

$$
\left\{\begin{array}{l}
T_{e}=q+T, \\
q_{e}=q-\alpha T-\hat{q},
\end{array}\right.
$$

and to rewrite (1.1) as

$$
\left\{\begin{array}{l}
\mathbf{u}_{t}+\beta(y) \mathbf{u}^{\perp}=(1+\alpha)^{-1}\left(\nabla T_{e}+\nabla q_{e}\right), \\
T_{e, t}-(1-\bar{Q}) \operatorname{div} \mathbf{u}=0, \quad \text { in } \quad \mathbb{R}^{2} \times(0, \infty), \\
q_{e, t}+(\bar{Q}+\alpha) \operatorname{div} \mathbf{u}=-\varepsilon^{-1}(1-\alpha) \phi\left(q_{e}\right) .
\end{array}\right.
$$

We continue stating some basic energy estimates for the solutions of (0.1). In what follows to emphasize the dependence of the solutions of $(0.1)$ on $\varepsilon$, we write $U^{\varepsilon}=\left(\mathbf{u}^{\varepsilon}, T^{\varepsilon}, q^{\varepsilon}\right)$ for the solution of (0.1). The independent of $\varepsilon$ energy estimates on $U^{\varepsilon}$ and its first spatial derivative $D U^{\varepsilon}$ were already established in $[\mathrm{FMP}]$, while the one for $U_{t}^{\varepsilon}$ follows in exactly the same way. Finally the estimate for $q_{e} \phi\left(q_{e}\right)$ is obtained after a straightforward integration using the information on $\left\|U^{\varepsilon}\right\|_{1}$ and $\left\|U_{t}^{\varepsilon}\right\|_{1}$, where $\|f\|_{0}$ and $\|f\|_{1}$ denote respectively the $L^{2}$ and $H^{1}$-norms in $\mathbb{R}^{2}$ of $f$. Note also that throughout the paper we denote by $C$ positive constants which depend on the data and may change from line to line.

Before we state the results we introduce the assumptions we need on the initial datum $U_{0}^{\varepsilon}$. It is worth mentioning that all the numerics done for $(0.1)$ use the same conditions.

Throughout the paper we assume that, for some $C>0$, which is independent of $\varepsilon$,

$$
\left\|\left(\mathbf{u}_{0}^{\varepsilon}, T_{0}^{\varepsilon}, q_{0}^{\varepsilon}\right)\right\|_{1} \leq C,
$$

and

$$
\left\|P\left(q_{e, 0}\right)\right\|_{0}^{2}=\int\left(\varepsilon^{-1} \phi\left(q_{e, 0}\right)\right)^{2} d x \leq C,
$$

where

$$
q_{e, 0}=q_{0}-\alpha T_{0}-\hat{q}
$$

Note that (1.5) is equivalent to

$$
\int_{\left\{q_{e, 0}^{\varepsilon} \geq 0\right\}}\left(q_{e, 0}^{\varepsilon}\right)^{2} d x \leq C \varepsilon^{2} .
$$

The energy estimates following directly from $[\mathrm{FMP}]$ are:

Theorem 1.1. Assume (0.3), (0.6), (0.5), (1.4) and (1.5). For each $M>0$, there exists a positive constant $C=C(M)$ that depends on only the bounds in (1.4) and (1.5) such that

$$
\max _{0 \leq t \leq M}\left[\left\|\left(\mathbf{u}^{\varepsilon}, T^{\varepsilon}, q^{\varepsilon}\right)(\cdot, t)\right\|_{1}+\left\|\left(\mathbf{u}_{t}^{\varepsilon}, T_{t}^{\varepsilon}, q_{t}^{\varepsilon}\right)(\cdot, t)\right\|_{1}\right] \leq C,
$$


and

$$
\int_{0}^{M} \int q_{e}^{\varepsilon} \phi\left(q_{e}^{\varepsilon}\right) d x d t \leq C \varepsilon
$$

The $L^{2}$-contraction property of the solutions of $(0.1)$ is:

Theorem 1.2. Assume (0.3), (0.6), (0.5), (1.4) and (1.5) and, for $i=1,2$, let $U^{\varepsilon, i}=$ $\left(\mathbf{u}^{\varepsilon, i}, T^{\varepsilon, i}, q^{\varepsilon, i}\right)$ be the solution of (0.1) with initial datum $U_{0}^{\varepsilon, i}$. Then

$$
\left\|U^{\varepsilon, 1}(\cdot, t)-U^{\varepsilon, 2}(\cdot, t)\right\|_{0} \leq\left\|U_{0}^{\varepsilon, 1}-U_{0}^{\varepsilon, 2}\right\|_{0} .
$$

Proof. Using the notation

$$
\left\{\begin{aligned}
\Delta \mathbf{u} & =\mathbf{u}^{\varepsilon, 2}-\mathbf{u}^{\varepsilon, 1}, \quad \Delta \mathbf{u}^{\perp}=\mathbf{u}^{\varepsilon, 2, \perp}-\mathbf{u}^{\varepsilon, 1, \perp}, \\
\Delta T & =T^{\varepsilon, 2}-T^{\varepsilon, 1}, \quad \Delta q=q^{\varepsilon, 2}-q^{\varepsilon, 1}, \quad \text { and } \quad \Delta P=P^{\varepsilon, 2}-P^{\varepsilon, 1}
\end{aligned}\right.
$$

we obtain

$$
\left\{\begin{array}{l}
(\Delta \mathbf{u})_{t}+\beta(y) \Delta \mathbf{u}^{\perp}=\nabla \Delta T, \\
(\Delta T)_{t}-\operatorname{div} \Delta \mathbf{u}=\Delta P \quad \text { in } \quad \mathbb{R}^{2} \times(0, \infty), \\
(\Delta q)_{t}+\bar{Q} \operatorname{div} \Delta \mathbf{u}=-\Delta P .
\end{array}\right.
$$

Finally arguing as in $[\mathrm{FMP}]$, we get, always in $\mathbb{R}^{2} \times[0, M]$,

$$
\left\{\begin{array}{l}
(\Delta q+\bar{Q} \Delta T)_{t}=-(1-\bar{Q}) \Delta P, \\
(\Delta q-\alpha \Delta T)_{t}+(\bar{Q}+\alpha) \operatorname{div}(\Delta \mathbf{u})=-(1+\alpha) \Delta P, \\
(\Delta q+\bar{Q} \Delta T)_{t}^{2}=-2(1-\bar{Q}) \Delta P(\Delta q+\bar{Q} \Delta T), \\
{\left[(\Delta T)^{2}+(\Delta \mathbf{u})^{2}\right]_{t}=2 \Delta T \Delta P .}
\end{array}\right.
$$

Adding the equations in (1.11) and integrating over $\mathbb{R}^{2}$, we find

$$
\left\{\begin{array}{l}
{\left[\int\left((\Delta T)^{2}+|\Delta \mathbf{u}|^{2}+((1-\bar{Q})(\alpha+\bar{Q}))^{-1}(\Delta q+\bar{Q} \Delta T)^{2}\right) d x\right]_{t}} \\
=2 \int\left(\left(\Delta T-(\alpha+\bar{Q})^{-1}(\Delta q+\bar{Q} \Delta T)\right) \Delta P\right) d x \\
=-(\alpha+\bar{Q})^{-1} \int(\Delta P(\Delta q-\alpha \Delta T)) d x \leq 0 .
\end{array}\right.
$$

The last inequality is due to the fact that, in view of the assumption on the source term $P$, we have

$$
\Delta P(\Delta q-\alpha \Delta T))=\varepsilon^{-1}\left[\phi\left(q_{e}^{\varepsilon, 2}\right)-\phi\left(q_{e}^{\varepsilon, 1}\right)\right]\left(q_{e}^{\varepsilon, 2}-q_{e}^{\varepsilon, 1}\right) \geq 0 .
$$

The claim now follows using (0.6). 


\section{EXISTENCE OF WEAK SOLUTIONS AT THE ZERO-RELAXATION Limit}

We study here the zero-relaxation limit, $\varepsilon \rightarrow 0$, of $(0.1)$ using the estimates of Theorem 1.1. Since, for any $t_{1}, t_{2} \in[0, M]$, there exists $C_{M}$ such that

$$
\left\|\left(\mathbf{u}^{\varepsilon}, T^{\varepsilon}, q^{\varepsilon}\right)\left(\cdot, t_{1}\right)-\left(\mathbf{u}^{\varepsilon}, T^{\varepsilon}, q^{\varepsilon}\right)\left(\cdot, t_{2}\right)\right\|_{0} \leq C_{M}\left|t_{1}-t_{2}\right|,
$$

the classical Lions-Aubin Lemma (see $[\mathrm{MB}]$ ) yields that, along subsequences which we denote the same way as the whole family, $\varepsilon \rightarrow 0$,

$$
\left(\mathbf{u}^{\varepsilon}, T^{\varepsilon}, q^{\varepsilon}\right) \rightarrow(\mathbf{u}, T, q) \quad \text { weakly in } C\left([0, M] ;\left(H^{1}\left(\mathbb{R}^{2}\right)\right)^{3}\right) \quad \text { and } \quad \operatorname{Lip}\left([0, M] ;\left(L^{2}\left(\mathbb{R}^{2}\right)\right)^{3}\right) \text {, }
$$

and, for any compactly supported $\rho \in C^{\infty}\left(\mathbb{R}^{2}\right)$,

$$
\rho\left(\mathbf{u}^{\varepsilon}, T^{\varepsilon}, q^{\varepsilon}\right) \rightarrow \rho(\mathbf{u}, T, q) \quad \text { strongly in } C\left([0, M] ;\left(H^{s}\left(\mathbb{R}^{2}\right)\right)^{3}\right) \quad \text { for all } s \in[0,1) .
$$

It is then immediate that, along subsequences $\varepsilon \rightarrow 0$,

$$
q_{e}^{\varepsilon} \rightarrow q_{e} \quad \text { weakly in } C\left([0, M] ; H^{1}\left(\mathbb{R}^{2}\right)\right) \times \operatorname{Lip}\left([0, M] ; L^{2}\left(\mathbb{R}^{2}\right)\right),
$$

and, for any compactly supported $\rho \in C^{\infty}\left(\mathbb{R}^{2}\right)$,

$$
\rho q_{e}^{\varepsilon} \rightarrow \rho q_{e} \quad \text { strongly in } C\left([0, M] ; H^{s}\left(\mathbb{R}^{2}\right)\right) \quad \text { for all } s \in[0,1) .
$$

The first observation is the following constraint for the limit $q_{e}$ of the $q_{e}^{\varepsilon}$ 's.

Proposition 2.1. Assume the hypotheses of Theorem 1.1. Then any limit $q_{e}$ of the $q_{e}^{\varepsilon}$ 's satisfies $q_{e} \leq 0$ a.e. in $\mathbb{R}^{2} \times(0, \infty)$.

Proof. Fix $M>0$. It follows from Theorem 1.7 and the form of $\phi$ that there exists $C>0$ such that, for each $R>0$,

$$
\int_{0}^{M} \int_{|x| \leq R} q_{e}^{\varepsilon} \phi\left(q_{e}^{\varepsilon}\right) d x d t \leq C \varepsilon .
$$

Letting $\varepsilon \rightarrow 0$ and using (2.4) we find

$$
\int_{0}^{M} \int_{|x| \leq R} q_{e} \phi\left(q_{e}\right) d x d t \leq 0,
$$

which, in view of (0.5), yields the claim.

Next we observe that the energy estimates and Proposition 2.1 also yield that $U=$ $(\mathbf{u}, T, q)$ assumes the initial data $U_{0}=\left(\mathbf{u}_{0}, T_{0}, q_{0}\right)$, and, moreover, in view of $(1.5), q_{e, 0}=$ $q_{0}-\alpha T_{0} \leq 0$ a.e. in $\mathbb{R}^{2}$.

We derive now the variational inequality satisfied by $U$. The result is: 
Theorem 2.1. Assume the hypotheses of Theorem 1.7 and let $(\mathbf{u}, T, q)$ be any limit, as $\varepsilon \rightarrow 0$, of $\left(\mathbf{u}^{\varepsilon}, T^{\varepsilon}, q^{\varepsilon}\right)$. Then, in $\mathbb{R}^{2} \times(, \infty)$,

$$
\left\{\begin{array}{c}
\mathbf{u}_{t}+\beta(y) \mathbf{u}^{\perp}=\nabla T \\
(q+T)_{t}-(1-\bar{Q}) \operatorname{div} \mathbf{u}=0, \\
q_{e, t}+(\bar{Q}+\alpha) \operatorname{div} \mathbf{u} \leq 0, \\
q_{e} \leq 0,
\end{array}\right.
$$

and

$$
q_{e, t}+(\bar{Q}+\alpha) \text { divu }=0 \quad \text { a.e. in } \quad\left\{q_{e}<0\right\} .
$$

Proof. It is immediate that (2.5) holds, while (2.7) is the assertion of Proposition 2.1 and (2.2) and (2.8) are immediate from the equation

$$
q_{e, t}^{\varepsilon}+(\bar{Q}+\alpha) \operatorname{divu}=-(1+\alpha) P^{\varepsilon} \leq 0 .
$$

Note that (2.2), (2.7) and (2.8) are written in the classical variational formulation following $[\mathrm{KS}]$. The information carried by $(2.2),(2.7)$ and (2.8) can also be expressed as in the following proposition.

Proposition 2.2. Assume the hypotheses of Theorem 1.7, fix $M>0$ and let $(\mathbf{u}, T, q)$ be any limit of $\left(\mathbf{u}^{\varepsilon}, T^{\varepsilon}, q^{\varepsilon}\right)$. Then, for all $\psi \in L^{2}\left(\mathbb{R}^{2} \times[0, M]\right)$ such that $\psi \leq 0$ a.e.,

$$
\int_{0}^{M} \int\left(q_{e, t}-(\bar{Q}+\alpha) d i v \mathbf{u}\right)\left(q_{e}-\psi\right) d x d t \leq 0 .
$$

Proof. Using that

$$
q_{e, t}^{\varepsilon}+(\bar{Q}+\alpha) \operatorname{div} \mathbf{u}^{\varepsilon}=-(1+\alpha) P^{\varepsilon},
$$

for any $\psi \in L^{2}\left(\mathbb{R}^{2}\right)$, we have

$$
\int_{0}^{M} \int\left(q_{e, t}^{\varepsilon}-(\bar{Q}+\alpha) \operatorname{div} \mathbf{u}\right)\left(q_{e}^{\varepsilon}-\psi\right) d x d t=-(1-\alpha) \int_{0}^{M} \int P^{\varepsilon}\left(q_{e}^{\varepsilon}-\psi\right) d x d t .
$$

But

$$
\begin{array}{r}
\int_{0}^{M} \int P^{\varepsilon}\left(q_{e}^{\varepsilon}-\psi\right) d x d t=\varepsilon^{-1} \int_{0}^{M} \int \phi\left(q_{e}^{\varepsilon}\right)\left(q_{e}^{\varepsilon}-\psi\right) d x d t= \\
=\varepsilon^{-1} \int_{0}^{M} \int_{\left\{q_{e}^{\varepsilon} \leq 0\right\}} \phi\left(q_{e}^{\varepsilon}\right)\left(q_{e}^{\varepsilon}-\psi\right) d x d t+\varepsilon^{-1} \int_{0}^{M} \int_{\left\{q_{e}^{\varepsilon} \geq 0\right\}} \phi\left(q_{e}^{\varepsilon}\right)\left(q_{e}^{\varepsilon}-\psi\right) d x d t .
\end{array}
$$


Since, for any nonnegative $\psi, q_{e}^{\varepsilon}-\psi \geq 0$ in $\left\{q_{e}^{\varepsilon} \geq 0\right\}$ and $\phi(r)=$ if $r \leq 0$,

$$
\int_{0}^{M} \int P^{\varepsilon}\left(q_{e}^{\varepsilon}-\psi\right) d x d t \geq 0,
$$

and the conclusion follows from (2.4).

\section{UNIQUENESS OF WEAK SOLUTIONS OF THE VARIATIONAL INEQUALITY}

We prove here that weak solutions of the variational inequality $(2.5),(2.2),(3.1)$ and (2.8) are unique.

We have:

Theorem 3.1. For any $U_{0} \in\left(H^{1}\left(\mathbb{R}^{2}\right)\right)^{3}$ such that $q_{0}-\alpha T_{0} \leq 0$ in $\mathbb{R}^{2}$, there exists at most one solution $\left.U \in\left(H^{1}\left(\mathbb{R}^{2}\right) \times[0, M]\right)\right)^{3} \bigcap C\left([0, M] ;\left(H^{1}\left(\mathbb{R}^{2}\right)\right)^{3}\right) \bigcap \operatorname{Lip}\left([0, M] ;\left(L^{2}\left(\mathbb{R}^{2}\right)\right)^{3}\right)$, for each $M>0$, of (2.5), (2.2), (2.7) and (2.8).

For the proof of Theorem 3.1 we need a result similar to Proposition 2.2, the only difference being the fact that here is derived it directly for weak solutions of $(2.5),(2.2),(2.7)$ and (2.8).

We have:

Proposition 3.1. Fix $M>0$ and let $\left(\mathbf{u}, q_{e}\right) \in\left(H^{1}\left(\mathbb{R}^{2}\right)\right)^{2}$ be a solution of $(2.5),(2.2)$, (2.7) and (2.8). Then, for $\psi \in L^{2}\left(\mathbb{R}^{2} \times[0, M]\right)$ such that $\psi \leq 0$ a.e.,

$$
\int_{0}^{M} \int\left(q_{e, t}+(\bar{Q}+\alpha) \operatorname{div} \mathbf{u}\right)\left(q_{e}-\psi\right) d x d t \leq 0 .
$$

Proof. The claim follows immediately from the observation that, if $F \in H^{1}\left(\mathbb{R}^{2} \times[0, M]\right)$ satisfies, for some $G \in L^{2}\left(\mathbb{R}^{2} \times[0, M]\right)$,

$$
F_{t}+G \leq 0 \quad \text { a.e. in } \quad \mathbb{R}^{2} \times[0, M] \quad \text { and } \quad F_{t}+G=0 \quad \text { in }\{F<0\},
$$

then, for any $\psi \in L^{2}\left(\mathbb{R}^{2} \times[0, M]\right)$ such that $\psi \leq 0$ a.e.,

$$
\int_{0}^{M} \int\left(F_{t}+G\right)(F-\psi) d x d t \leq 0 .
$$

Indeed, if $\chi_{A}$ denotes the characteristic function of the set $A$,

$$
\int_{0}^{M} \int\left(F_{t}+G\right)(F-\psi) d x d t=\int_{0}^{M} \int\left(F_{t}+G\right)\left[\chi_{\{F \geq 0\}}(F-\psi)+\chi_{\{F<0\}}(F-\psi)\right] d x d t .
$$

Since $\chi_{\{F \geq 0\}}(F-\psi) \geq 0$ (recall that $\psi$ is non positive), the claim for $F$ follows from (3.2), and, hence, (3.1) holds. 
We proceed now with the

Proof of Theorem 3.1. Fix $M>0$ and let $U^{1}, U^{2} \in\left(H^{1}\left(\mathbb{R}^{2} \times[0, M]\right)\right)^{3} \cap C\left([0, M] ;\left(H^{1}\left(\mathbb{R}^{2}\right)\right)^{3}\right) \cap \operatorname{Lip}([0, M]$; be two solutions of (2.5), (2.2), (3.1) and (2.8) with the same initial data.

In view of Proposition 3.1, for any non positive $\psi \in L^{2}\left(\mathbb{R}^{2} \times[0, M]\right)$, we have, for $i=1,2$,

$$
\int_{0}^{M} \int\left(q_{e, t}^{i}+(\bar{Q}+\alpha) \operatorname{div} \mathbf{u}^{i}\right)\left(q_{e}^{i}-\psi\right) d x d t \leq 0
$$

and

$$
\int_{0}^{M} \int\left(\left(q_{e}^{i}+T^{i}\right)_{t}-(1-\bar{Q}) \operatorname{div} \mathbf{u}^{i}\right)\left(q_{e}^{i}-\psi\right) d x d t=0
$$

Multiplying (3.4) by $1-\bar{Q}$ and (3.5) by $\bar{Q}+\alpha$ and adding the resulting inequalities we find, for $i=1,2$,

$$
\int_{0}^{M} \int\left((\bar{Q}+\alpha)\left(q^{i}+T^{i}\right)_{t}+(1-\bar{Q})\left(q^{i}-\alpha T^{i}-\hat{q}\right)\right)_{t}\left(q_{e}^{i}-\psi\right) d x d t \leq 0
$$

and, hence,

$$
\int_{0}^{M} \int\left(q^{i}+\bar{Q} T^{i}\right)_{t}\left(q_{e}^{i}-\psi\right) d x d t \leq 0
$$

Using in (3.6) $\psi=q_{e}^{2}$ if $i=1$ and $\psi=q_{e}^{1}$ if $i=2$ and adding the resulting two inequalities we get

$$
\int_{0}^{M} \int\left(\left(q^{2}-q^{1}\right)+\bar{Q}\left(T^{2}-T^{1}\right)\right)_{t}\left(\left(q^{2}-q^{1}\right)-\alpha\left(T^{2}-T^{1}\right)\right) d x d t \leq 0 .
$$

Observe next that, if $\Delta q=q^{2}-q^{1}$ and $\Delta T=T^{2}-T^{1}$, then

$$
\begin{array}{r}
(\Delta q+\bar{Q} \Delta T)(\Delta q+\bar{Q} \Delta T)_{t}=(\Delta q-\alpha \Delta T)(\Delta q+\bar{Q} \Delta T)_{t}+ \\
((\Delta q+\bar{Q} \Delta T)-(\Delta q-\alpha \Delta T))(\Delta q+\bar{Q} \Delta T)_{t}
\end{array}
$$

Hence, in view of (3.7),

$$
\begin{array}{r}
\int_{0}^{M} \int(\Delta q+\bar{Q} \Delta T)_{t}^{2} d x d t \leq 2(\bar{Q}+\alpha) \int_{0}^{M} \int(\Delta q+\bar{Q} \Delta T)_{t} \Delta T d x d t= \\
2 \int_{0}^{M} \int\left((\bar{Q}+\alpha)(\Delta q+\Delta T)_{t} \Delta T+(\bar{Q}-1) \Delta T(\Delta T)_{t}\right) d x d t
\end{array}
$$

and, finally,

$$
\int_{0}^{M} \int\left((\Delta q+\bar{Q} \Delta T)^{2}+(1-\bar{Q})(\Delta T)^{2}\right)_{t} d x d t \leq 2(\bar{Q}+\alpha) \int_{0}^{M} \int(\Delta Q+\Delta T)_{t} \Delta T d x d x t
$$


However, since

$$
(\Delta q+\Delta T)_{t}-(1-\bar{Q}) \operatorname{div} \Delta \mathbf{u}=0
$$

we get

$$
\int_{0}^{M} \int(\Delta q+\Delta T)_{t} \Delta T d x d t=(1-\bar{Q}) \int_{0}^{M} \int \operatorname{div}(\Delta \mathbf{u}) \Delta T d x d t
$$

Combining (3.8) and (3.9) we find

$$
\int_{0}^{M} \int\left((\Delta q+\bar{Q})^{2}+(1-\bar{Q})(\Delta T)^{2}\right)_{t} d x d t \leq 2(\bar{Q}+\alpha)(1-\bar{Q}) \int_{0}^{M} \int \operatorname{div}(\Delta \mathbf{u}) \Delta T d x d t .
$$

Finally, since

$$
\int_{0}^{M} \int(\Delta U)_{t} \cdot \Delta U d x d t=\int_{0}^{M} \int \nabla \Delta T \cdot \Delta U d x d x t
$$

we obtain

$$
\int_{0}^{M} \int\left((\Delta q+\bar{Q})^{2}+(1-\bar{q})(\Delta T)^{2}+(\bar{Q}+\alpha)(1-\bar{Q})|\Delta U|^{2}\right)_{t} d x d t \leq 0
$$

The uniqueness now follows in view of (0.6).

\section{Concluding Remarks}

In Sections 2 and 3 we established the existence and uniqueness of weak solutions to a novel hyperbolic free boundary problem arising in tropical meteorology. Clearly the arguments can also be used to study the same system in $N$-space dimensions. It is worth mentioning that the hypotheses on the rotation coefficient $\beta(y)$ were utilized to avoid subtle issues at infinity. Weakening (0.3) could require the use of special weighted Sobolev spaces in the limiting estimates ([DMS]). There is (see [FMP]) an interesting more complex version of (0.1) involving coupling with the barotropic model. Obtaining higher order energy estimates for this problem is a hard unsolved problem. The models discussed here are excellent ones for understanding the precipitation fronts at large scales in GCM's. The actual behavior as observed in nature is captured in a much more realistic fashion by more complex multi-cloud models [KM3, KM4] with large scale instability.

\section{REFEREnCES}

[DMS] A. Dutrifoy, A. J. Majda and S. Schochet, A simple justification of the singular limit for equatorial shallow-water dynamics, Comm. in Pure and Applied Math. LXI (), 2-12.

[FMP] D. M. W. Frierson, A. J. Majda and O. Pauluis, Large scale dynamics of precipitating fronts in the tropical atmopsphere: A novel relaxation limit, Commun. Math. Sci 19 (2004), 591-626.

[KS] D. Kinderlehrer and G. Stampacchia, An introduction to variational inequalities and their applications, Academic Press, New York, 1980.

[KM1] B. Khouider and A. J. Majda, A non-oscillatory balanced scheme for an idealized tropical climate model. Part I: Algorithm and validation, Theor. Comput. Fluid Dyn. 19 (2005), 331-354. 
[KM2] B. Khouider and A. J. Majda, A non-oscillatory balanced scheme for an idealized tropical climate model. Part II: Nonlinear coupling and moisture effects, Theor. Comput. Fluid Dyn. 19 (2005), $355-375$.

[KM3] B. Khouider and A. J. Majda, A Simple Multi-Cloud Model for Convectively Coupled Tropical Waves. Part I: Linear Analysis. J. Atmos. Sci. 63 (2006), 1308-1323.

[KM4] B. Khouider and A. J. Majda, A Simple Multi-Cloud Parameterization for Convectively Coupled Tropical Waves. Part II: Nonlinear Simulations. J. Atmos. Sci. 64 (2007), 381-400.

[M] A. J. Majda, Compressible fluid flow and systems of conservation laws in several space variables, Applied Math Sci., Vol 53, Springer-Verlag, New York 1984.

[MB] A. J. Majda and A. Bertozzi, Vorticity and Incompressible Flow, (2002) Cambridge University Press, United Kingdom.

[PFM] O. Pauluis, D. M. W. Frierson and A. J. Majda, Preipitation fronts and the reflection and transmissions of tropical disturbances, Q. J. R. Meteorol. Soc. 134 (2008), 913-930.

[SM] S. N. Stechmann and A. J. Majda, The structure of precipitation fronts for finite relaxation time, Theor. Comput. Fluid Dyn. 20 (2006), 377-404. 